Testing Large CICC in Short Sample Configuration and Predicting Their Performance in Large Magnets

N. N. Martovetsky

August 29, 2007

IEEE Transactions on Applied Superconductivity 
This document was prepared as an account of work sponsored by an agency of the United States Government. Neither the United States Government nor the University of California nor any of their employees, makes any warranty, express or implied, or assumes any legal liability or responsibility for the accuracy, completeness, or usefulness of any information, apparatus, product, or process disclosed, or represents that its use would not infringe privately owned rights. Reference herein to any specific commercial product, process, or service by trade name, trademark, manufacturer, or otherwise, does not necessarily constitute or imply its endorsement, recommendation, or favoring by the United States Government or the University of California. The views and opinions of authors expressed herein do not necessarily state or reflect those of the United States Government or the University of California, and shall not be used for advertising or product endorsement purposes. 


\title{
Testing large CICC in short sample configuration and predicting their performance in large magnets
}

\author{
Nicolai Martovetsky
}

\begin{abstract}
It is well known that large Nb3Sn Cable-in-Conduit Conductors (CICC) do not always completely utilize current carrying capacity of the strands they are made of. The modern state of theory is not accurate enough to eliminate CICC full scale testing. Measuring properties of large CICC is not a simple task due to variety of parameters that need to be controlled, like temperature, exposure of all the strands to the peak magnetic field, mass flow and particular nonuniform current distribution.

The paper presents some measurement issues of CICC testing in a short sample test facility, particularly, conditions for uniform current distribution and effect of twist pitches on the critical current.
\end{abstract}

Index Terms-Current distribution, electric field measurement, magnetic field, superconducting cables.

\section{INTRODUCTION}

$\mathrm{Q}$ UALIFICATION of ITER Cable in-Conduit Conductors (CICC) is a very important step for reliable achievement of ITER design parameters. The most economical way of testing the conductors is testing a short sample in the SULTAN facility [1] in Switzerland.

Recent testing campaigns [2] showed that the measurements of the short samples do not give clean transitions of a superconductor in the normal state that would allow an easy interpretation of current sharing temperature Tcs. A common feature of many test results was the appearance of a significant voltage before exponential transition became observable and strong deviation from a typical exponential growth of voltage versus temperature or current.

Most analysts believe that such signals come from nonuniform current distribution of the conductor in the cable due to unavoidable scatter of resistances between the strands and terminations. Indeed, before strands develop resistance, the current distribution is dictated by the strand resistances to the terminals, and when superconducting strands develop some resistance, a redistribution of current starts. The effects of nonuniform current distribution and signal processing

Manuscript received August 28, 2007. This work was supported by the US Department of Energy under contract to UC, Lawrence Livermore National Laboratory (contract No. W-7405-Eng-48).

The author is with Lawrence Livermore National Laboratory, Livermore, currently on assignment to US ITER Project Office, at ORNL, 1055 Commerce Park, Oak Ridge, TN, 37830 approaches to deduce a performance for uniform current distribution are discussed in [3]. It is shown that there is no reliable way to project performance of the uniform current distribution from the test data obtained on a conductor with a nonuniform current distribution, therefore the sample should be prepared to reduce or eliminate effects of nonuniform current distribution. In this paper we discuss conditions that could assure uniform current distribution.

The question is how low the transverse resistance is sufficient in order to provide a more or less uniform current distribution. ITER magnets and other magnets with a long conductor length in the magnetic field have uniform current distribution in the cable and therefore performance of a CICC at the uniform current distribution is our primary interest.

\section{CONDITIONS FOR UNIFORM CURRENT Distribution}

Let's start with the simplest two wire model shown in Fig. 1 and analyze ability of current redistribution. The Rsc1 and Rsc2 represent resistances of superconducting wires and $\mathrm{Rp}$ are transverse resistances. The voltage at which the temperature of current sharing (Tcs) is determined in SULTAN is $4.5 \mu \mathrm{V}$ (comes from $10 \mu \mathrm{V} / \mathrm{m}$ criterion and 0.45 $\mathrm{m}$ long length in high magnetic field). Let us estimate a maximum nonuniformity in the schematic of Fig. 1. Obviously the worst nonuniformity can occur when $\mathrm{Rsc} 2=0$. At I1 Rsc $1=4.5 \mu \mathrm{V}$ the transverse current that can be pushed from wire 1 into wire 2 is $\Delta \mathrm{I}=4.5 \mu \mathrm{V} /(2 \mathrm{Rp})$. In the ITER $\mathrm{TF}$ conductor each superconducting strand carries about $70 \mathrm{~A}$ in operation. If the ability to transfer the current is $\geq 70 \mathrm{~A}$ at this voltage, it is guaranteed that the current at this voltage is uniformly distributed to within a parameter Io, where Io is the increment of the electrical field growth versus current. When current in a strand grows by Io, the electrical field growths by a factor of $\mathrm{e}=2.72 \ldots$ To obtain such a uniformity we need to

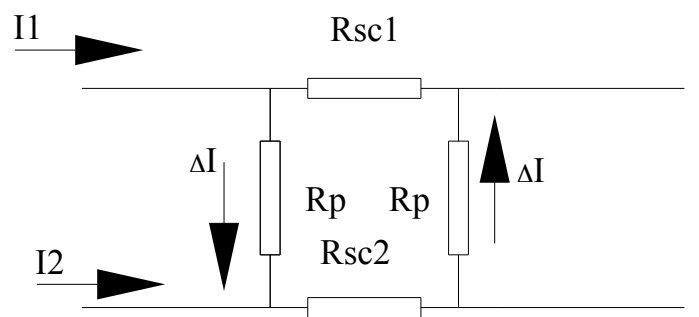

Fig.1. Two wire simple model 
have $\mathrm{Rp}=32 \mathrm{nOhm}$ or better.

Now let's imagine that in a 1000 strands cable we measure strand to strand resistance of $10 \mathrm{nOhm}$. Is it sufficient to guarantee a uniform distribution at $4.5 \mu \mathrm{V}$ in some strands?

On the first glance it looks like a comfortably low resistance for uniform distribution, but we should not forget that the resistance between the strands in a cable is very different from resistance between two strands when the other strands are not present.

In the cable each strand has multiple contacts with the other strands. Let's assume that each strand is in contact with all other strands in say, 1000 strand cable. Then, when we apply a voltage between two strands called $\mathrm{A}$ and $\mathrm{B}$ to measure resistance, the current will go from the A strand to every other strand and then to the B strand. Of course, there will be also a direct current path from $\mathrm{A}$ to $\mathrm{B}$ in addition to that. Thus the conductance between the strands A and B will be as follows:

$$
\sigma_{A B}=\sigma+999 \sigma / 2 \approx 500 \sigma
$$

where $\sigma$ is the conductance between the two strands, if there were extracted from the cable.

So, if we measured $10 \mathrm{nOhm}$ between two strands in the cable and each strand is connected to all the rest through some resistance, the actual resistance between two strands extracted from the cable would be 500 times higher. In our case the resistance between two strands extracted from the cable would be $5 \mu \mathrm{Ohm}$.

Let us now imagine that the joints will provide a nonuniform current distribution, with 500 strands carrying one current value and the rest 500 strands carrying a different current. In this case, the behavior of the cable would be equivalent to the situation in the two wire model, shown in Fig. 1, where the first group of strands will be under the same potential and the second group of strands will be under a different potential. The current will flow from one strand from the group of strands with higher current to another strand in the group with a smaller current. Due to the symmetry there will be no cross talking between the strands in one group carrying the same current. Then at $4.5 \mu \mathrm{V}$ and $5 \mu \mathrm{Ohm}$ the ability to transfer current from one strand to another in such a situation is less than $1 \mathrm{~A}$. Thus, in order to guarantee a uniform current distribution at $4.5 \mu \mathrm{V}$, the resistance between the strands in the cable should be lower than $10 \mathrm{nOhm}$. This example may have some exaggeration in the assumption that every strand has an immediate contact with every other strand. On a limited length it is unlikely that every strand is in the

E4



Fig.2. Two wire model of a SULTAN sample direct contact with all the others, which would lead to the resistance value between the strands somewhat lower and situation in reality could be somewhat better than in the example above, but we often do not know in advance how nonuniform the distribution of the current is in the cable.

Based on our observation, let us derive a qualitative criterion for uniform distribution in the two wire model, shown in Fig. 2. It represents a schematic of the current distribution between two wires. Let is assume that Rsc1 and Rsc2 are resistances of the superconducting transition in the wires and the voltage drop in each superconductor are V1 and $\mathrm{V} 2$, respectively. Some voltmeters are shown for a discussion below. For instance, the V2 is measured by the voltmeter E1.

The resistances $\mathrm{Rj} 1-\mathrm{Rj} 4$ represent joints and the scatter of these resistances will dictate the current distribution until resistances in superconductors start developing. Let's see if without transverse resistance the resistance in the superconducting strands is sufficient to ensure uniform current distribution. Let's suppose that the joint resistance is $1 \mathrm{nOhm}$ and voltmeters E2 and E3 read $68 \mu \mathrm{V}$ at the ITER TF operating current of $68 \mathrm{kA}$.

The resistance in the superconductor reaches the "critical" value of $4.5 \mu \mathrm{V}$ at $68 \mathrm{kA}$ in the CICC. Even for good joints the scatter in resistances between the strands [4] is $+/-20 \%$. Thus, the fact that the joints have a very low resistance (in a sense that it is difficult to obtain a better resistance) does not guarantee that the current distribution is uniform.

Due to a nonuniform current distribution the voltages in the superconductor start developing nonuniformly too. The current from the wire, which has a higher voltage drop is transferred partially to the other strand through transverse resistances in the joint (Rp1) or in the cable (Rp2).

Let's assume that the length of the joint is L1, and the transverse resistance per unit length is Rp1. The transverse resistance per unit length in the joints is usually significantly lower than the transverse resistance in the regular cable because the strands in the joint typically have significantly lower void fraction and the chrome coating is removed from the surface. In some cases the joints partially or fully filled with solder. The transverse resistance per unit length in the cable outside the joint is Rp2, and the length of the cable between the joint and the center of the magnetic field is L2.

When a nonuniform current generates a nonuniform voltage in the strands, the amount of current that could be transferred through $\mathrm{Rp} 1$ and $\mathrm{Rp} 2$ is:

$$
I_{\perp}=\frac{V\left(R_{p 1} L_{2}+R_{p 2} L_{1}\right)}{R_{p 1} R_{p 2}}
$$

If this current is comparable to the transport current in the wire, then despite a nonuniform distribution of current in the joints, the transverse resistance will assure a uniform current distribution in the high field area. Extending formula (2) to the case of the full-scale CICC we should use in (2) the measured resistances between two strands in the joint and in the cable, but the transverse current in (2) should be taken as about half of the total transport current in the cable to be conservative. 
Thus, at the voltage of $4.5 \mu \mathrm{V}$ and transverse current of $35 \mathrm{kA}$ the measured resistance between two strands should be of order of $0.13 \mathrm{nOhm}$ to ensure uniform current distribution in the cable. This is a sufficient criterion. If the original distribution of current is more uniform, the transverse resistance could be higher.

Let's compare the ability to transfer current inside the cable for two cases:1) a SULTAN short sample, and 2) the ITER TF magnet. In the TF magnet double pancake about $24 \mathrm{~m}$ of conductor is located in the peak field versus $0.45 \mathrm{~m}$ in the SULTAN sample. Thus at the same electrical field of 10 $\mu \mathrm{V} / \mathrm{m}$ the voltage in the conductor is $240 \mu \mathrm{V}$ in the TF versus $4.5 \mu \mathrm{V}$ in SULTAN sample.

In addition, the length L2 in the pancake is about $400 \mathrm{~m}$ versus $0.5-1 \mathrm{~m}$ in SULTAN. The combination of these parameters makes distribution of current much more uniform in the ITER magnet.

What can be done for the SULTAN sample preparation to ensure uniform current distribution in the cable?

From (2) we can see that the only practical approach is to reduce the transverse resistance in the joint. Increasing the voltage is not desirable in SULTAN, since it requires high electrical fields that can not be reached in ITER magnet or even in SULTAN. Changing resistance in the cable, the overall length of the cable or the length of the cable in the high field are all impractical options. If the nonuniform current distribution in SULTAN samples is not solved by joints with very little scatter of resistances, the only practical mitigation is to reduce the resistance $\mathrm{Rp} 1$ by removing $\mathrm{Cr}$ between the strands and solder filling the cable space. This process was tried on the TFAS1 sample [5]. This sample showed significant voltage developing from the very start of charging. It also showed significant deviation from the exponential growth of voltage versus temperature and current. An exponential growth of voltage versus current and temperature was observed in many experiments [6], including measurements on solenoid Inserts with long lengths of the conductor in uniform field that would assure a uniform current distribution. To reduce the interstrand resistance the joints in the TFAS1 conductors were solder filled.

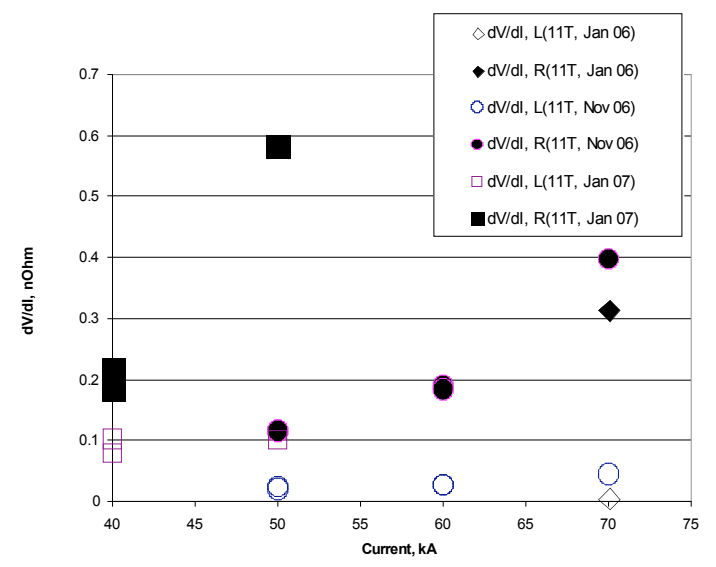

Fig.3. Evolution of the slope in TFAS1 sample

The results are given in [5], here we report only change of the slope of the voltage versus current. Fig. 3 shows measurements of the V-I slope in three different campaigns January and November 2006 and in January of 2007, after solder filling of the joint. It is clear that the slope increased significantly after the solder filling, which is exactly the opposite of the intent and expectation. Not only did the slope of the voltage increase, the overall resistance of the joints also increased significantly. For example, the resistance of the lower joint increased from 2 to $18 \mathrm{nOhm}$. Later de-sectioning of the terminations revealed good quality of filling with very few voids. This suggests that the interface resistance between the strands and the solder in the solder filled termination increased for some unknown reason and resulted in increases of both the total resistance and the interstrand resistance in the termination.

This experience shows that solder filling should be planned in advance and performed in well controlled conditions. There is no doubt that in an optimal solder filling joint the interstrand resistances will be lower than in a simply mechanically compressed termination. We are planning to solder fill the terminations that will have stripped chrome plating prior to the heat treatment. Obtaining low interstrand resistance is a very important task that may help to solve the current nonuniformity problem and establish SULTAN facility suitable for qualification of the ITER conductors.

\section{EfFect OF MAgnet Field Length AND Twist Pitches IN THE CICC IN ASSESSMENT OF THE SULTAN TEST RESUlTS}

Recent test results from one of a SULTAN sample, TFPRO-OST2 [7] with long twist pitches showed significantly lower degradation of that sample than similar cables with sharper twist pitches. It also showed a high $\mathrm{N}$-value and low deviation from an exponential transition.

The main reason for such behavior is thought to be a better support of the strands in the cable, where longer twist pitches provided line support to the strands instead of localized point contacts for the cables with shorter twist pitches.

The cable pattern is $((2 \mathrm{~s} / \mathrm{c}+1 \mathrm{Cu}) \times 3 \times 5 \times 5+$ core $)$; core: $3 \mathrm{x} 4 \mathrm{Cu}$. The twist pitches for the baseline and the test conductor TFPRO-OST2 are given in Table 1.

The last two stages of the cable are close to the length of the magnetic field in SULTAN. In this condition, some strands in the cable may not see the peak field in the cable space and because of that they will have a higher critical current Ic. This

TABLE 1 Twist PITCHES OF THE TF CABLE FOR BASELINE AND TFPRO-OST2 VERSIONS

\begin{tabular}{lll}
\hline \hline Stage & $\begin{array}{l}\text { Baseline twist } \\
\text { pitch } \\
\text { specification, mm }\end{array}$ & $\begin{array}{l}\text { Twist pitch } \\
\text { specification, } \\
\text { TFPRO- OST2, } \\
\text { mm }\end{array}$ \\
\hline Stage 1 & $45+/-5$ & 116 \\
Stage 2 & $85+/-8$ & 182 \\
Stage 3 & $125+/-10$ & 245 \\
Stage 4 & $250+/-15$ & 415 \\
Stage 5 & $450+/-45$ & 440
\end{tabular}


effect will make the current carrying capacity of the cable appear higher than it would be in the TF coil, where the length of the conductor is much longer and all strands will be exposed to a high magnetic field.

In order to estimate the effect of a long twist pitch on Ic of the CICC in SULTAN facility we developed a model of the cable. We computed magnetic and electrical fields in the strands and integrated electrical field from joint to joint in the SULTAN along many strands. We assumed that the cable had zero transverse resistances between the strands and that the currents in the strands are distributed according to the voltages generated due to superconducting transitions.

In our model we represent a strand as a multiple spiral in order to reproduce the cabling process. Only three last cable stages are taken into account, the first two stages are unimportant for space location of the spiral in the cable. Fig. 4 shows a coordinate y (projection on the plane based by the conductor centerlines) for five selected strands in the cable equally distanced from each other. The magnetic field depends linearly on this coordinate. In a well-transposed cable, the projection of a strand on a plane has characteristic features of all twist pitches of all spirals and the strand projection travel all the way from a maximum position of $20 \mathrm{~mm}$ at peak field to $-20 \mathrm{~mm}$ to the lowest field. Fig.4 shows that there is not much deviation from a pure sinusoidal shape, which suggests that the strands are not effectively transposed and may have problems with high losses and stability. Most important is that some strands are not exposed to the peak field. Fig. 5 shows distribution of the magnetic field in these strands.

As one can see, the maximum field for some strands in the SULTAN sample is much lower than the peak field for the conductor, in our case in Fig. 5 the peak field for some strands does not exceed $11.3 \mathrm{~T}$ at peak field of $12 \mathrm{~T}$. This means that Tcs for the TFPRO-OST2 conductor will be higher in SULTAN than in TF magnet operation by $0.3-0.5 \mathrm{~K}$.

There are several alternatives to qualify ITER conductors with longer twist pitch combinations. One approach is to take into account the artificial enhancement of Ic and allow an additional margin for the critical current. Another alternative is to use a facility that has a longer magnetic field. The third

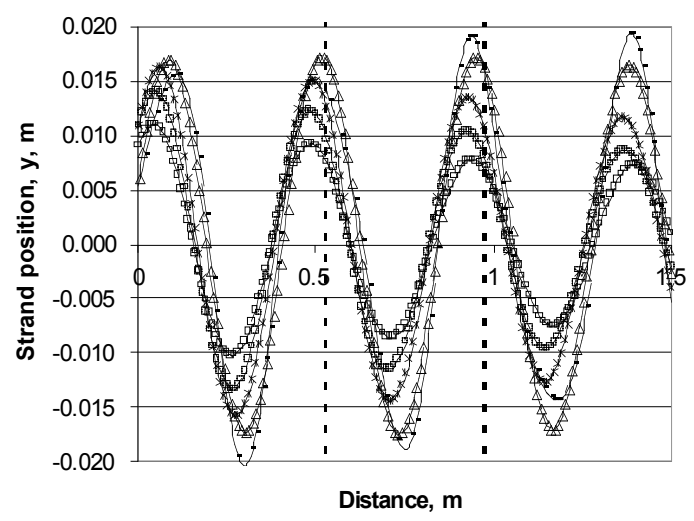

Fig.4. Projection of position of some selected strands in the TFPROOST2 cable. The vertical dot lines identify SULTAN peak field region.

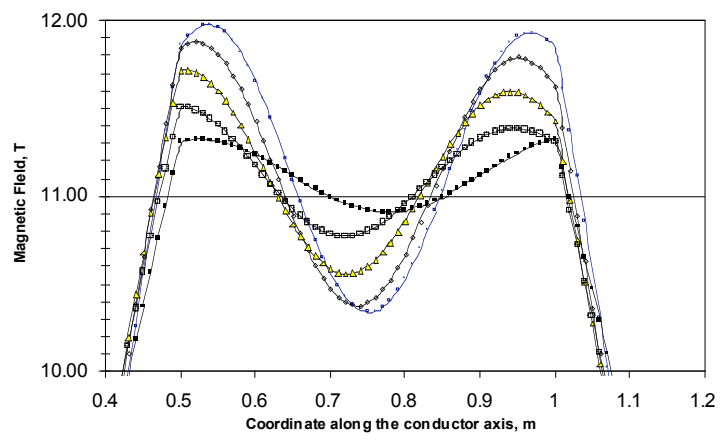

Fig. 5. Magnetic field distribution along selected strands in the cable

option is to maintain the design of the cable with shorter twist pitches that allow exposing all or most of the strands to the peak field.

\section{CONCLUSION}

Predicting performance of the CICC on the basis of a short sample requires a uniform current distribution at testing. The only practical approach to improve nonuniform distribution in a short sample is to reduce the interstrand resistance in the joint. Achieving a $1 \mathrm{nOhm}$ overall resistance in the joints may not be sufficient for uniform current distribution.

Short sample test facility may not expose all the strands to the peak field resulting in too optimistic results. Predicting performance of CICC in ITER magnets requires a correction for this effect or selection of the cabling pattern appropriate for short sample test facility.

\section{ACKNOWLEDGMENT}

Author is thankful to J. Miller for valuable discussions and to $\mathrm{W}$. Meier for his interest and useful suggestions.

\section{REFERENCES}

[1] P. Bruzzone, A. Anghel, A. Fuchs, G. Pasztor, B. Stepanov, M. Vogel and G. Vecsey, "Upgrade of Operating Range for SULTAN Test Facility", IEEE Trans. Applied Superconductivity, vol. 12, No. 1, March 2002, pp. 520-523.

[2] Session 4I, this conference

[3] N. Martovetsky, "Testing short samples of ITER conductors and projection of their performance in ITER magnets", presented at 5th Annual Workshop on Mechanical and Electromagnetic Properties of Composite Superconductors - MEM '07, Princeton, 21-24 August, 2007

[4] P. Bruzzone, B. Stepanov, R. Dettwiler, F. Staehli "Results of Contact Resistance Distribution in $\mathrm{NbTi}$ and $\mathrm{Nb} 3 \mathrm{Sn}$ ITER Conductor Termination", IEEE Appl. Supercond., vol. 17, No. 2, June 2007, p.1378

[5] Stepanov, P. Bruzzone, R. Wesche, M. Bagnasco, M. Vogel, D. Ciazynski, N.N. Martovetsky, "Retest of the TFAS1 sample with solder filled extremities", this conference

[6] N. Martovetsky, "Measurements of Ic and Tcs in CICC: assumptions and interpretations", presentation at ITER Nb3Sn Conductor Modeling Workshop 15-17 January 2007, unpublished.

[7] P. Bruzzone, B. Stepanov, R. Wesche, E. Salpietro, A. Vostner, K.K. Okuno, T. Isono, H.C. Kim, K. Kim, A. Shikov, V. Pantsyrny, "Results of a new generation of ITER TF conductor samples in SULTAN", this conference 